\title{
Variation of Radio Refractivity with Respect to Moisture and Temperature and Influence on Radar Ray Path
}

\author{
Jidong GAO*1, Keith BREWSTER ${ }^{1}$, and Ming XUE ${ }^{1,2}$ \\ ${ }^{1}$ Center for Analysis and Prediction of Storms \\ ${ }^{2}$ School of Meteorology, University of Oklahoma, Norman, Oklahoma 73072, USA
}

(Received 16 August 2007; revised 11 March 2008)

\begin{abstract}
In this study, the variation of radio refractivity with respect to temperature and moisture is analyzed. Also, the effects of vertical gradients in temperature and moisture on the propagation paths of electromagnetic waves of weather radar are examined for several sites across the United States using several years of sounding data from the National Weather Service. The ray path is important for identifying storm characteristics and for properly using the radar data in initializing numerical weather prediction models. It is found that during the warm season the radio refractivity gradient is more sensitive to moisture gradients than to temperature gradients. Ray paths from the commonly accepted vertical ray path model are compared to a ray path computed from a stepwise ray tracing algorithm using data from actual soundings. For the sample of about 16000 soundings examined, we find that only a small fraction of the ray paths diverge significantly from those calculated using a ray path model based on the US Standard Atmosphere. While the problem of ray ducting in the presence of a temperature inversion is fairly well known, we identify the presence of a strong vertical moisture gradient as the culprit in the majority of the cases where significant deviations occurred.
\end{abstract}

Key words: radio refractivity, radar ray path

Citation: Gao, J. D., K. Brewster, and M. Xue, 2008: Variation of radio refractivity with respect to moisture and temperature and influence on radar ray path. Adv. Atmos. Sci., 25(6), 1098-1106, doi: 10.1007/s00376-008-1098-x.

\section{Introduction}

The United States operational WSR-88D Doppler radar network (NEXRAD) is a vital tool for the real-time detection and warning of hazardous weather (Crum and Albert, 1993; Crum et al., 1998; Serafin and Wilson, 2000). It is also an essential observing system for initializing non-hydrostatic, storm-resolving (i.e., horizontal grid spacing on the order of $1 \mathrm{~km}$ ) numerical weather prediction (NWP) models (e.g., Lilly, 1990; Droegemeier, 1990, 1997). Attempts to demonstrate such capability began early in the past decade (e.g., Sun et al., 1991), and subsequent efforts have been notably successful (e.g., Gao et al., 1998; Sun and Crook, 2001; Weygandt et al., 2002a,b; Crook and Sun, 2002; Xue et al., 2003; Brewster, 2003; Gao et al., 2004; Hu et al., 2006a,b).

To utilize the radar reflectivity and radial veloc- ity data in real-time warning and quantitative precipitation estimation and to assimilate the data into NWP models, it is necessary to accurately determine the spatial locations of individual radar measurements. Because the propagation path of the electromagnetic waves can be affected by the refractivity of the atmosphere, the propagation path or the ray path is usually not a straight line. A suitable ray path equation is needed; the local direction of the path also affects the radial velocity forward operator that projects the Cartesian velocity components on the model grid to the local radial direction in data assimilation systems.

Most early radar data assimilation studies used relatively simple ray path equations in the forward operator formulation, with the simplest one being based on the Cartesian flat-earth geometry (e.g., Gao et al., 1998, 2004; Weygandt et al., 2002a,b; Shapiro et al., 2003). The next level of sophistication is to use the

\footnotetext{
${ }^{*}$ Corresponding author: Jidong GAO, jdgao@ou.edu
} 
four-thirds earth radius model (see, e.g., Doviak and Zrnic, 1993; Gao et al., 2006) for the radar ray path calculations (e.g., Brewster, 2003). This model takes into account the curvature of the earth but assumes that the atmosphere has a constant vertical gradient of refractivity in the lower troposphere as determined from the U.S. Standard Atmosphere. In reality, the gradient of the refractivity is seldom constant and significant departures from the assumption exist when there are strong temperature inversions and/or large vertical moisture gradients. A better understanding of the variation of the ray path due to the gradient of refractivity as well as a better understanding of the frequency of occurrence of significant departures from the path prediction of simple models is valuable to radar data quality control and quantitative precipitation estimation (Bech et al., 2003). Serious contamination of radar data can occur in atmospheric conditions that cause anomalous propagation of the radar beam (Moszkowicz et al., 1994; Pamment and Conway, 1998). In this study, the variation of radio refractivity with respect to gradients in temperature and moisture is first analyzed; the influence of atmospheric environmental conditions on the ray paths at locations representing four different climate regions of the United States is then examined using sounding data over several years from the US National Weather Service.

The rest of this paper is organized as follows: In section 2 , the four-thirds earth radius model for radar ray path calculations is briefly reviewed. An analysis of the variation of refractivity with respect to temperature and moisture is given in section 3 . In section 4 , a stepwise ray trace method is introduced. In section 5 , the influence of atmospheric refractivity on the ray path at different geographical locations in the United States is examined using historic sounding data from the National Weather Service. Finally, a summary and conclusions are given in section 6 .

\section{Ray path equations based on the four- thirds earth radius model}

Under the assumption that temperature and humidity are horizontally homogeneous, so that refractivity is a function of height above ground only, a formula can be derived (e.g., Doviak and Zrnic, 1993) that expresses the ray path in terms of a curve following a sphere of effective radius

$$
a_{e}=\frac{a}{1+a(d n / d h)}=k_{e} a,
$$

where $a$ is the earth's radius and $k_{e}$ is a multiplier that depends on the vertical gradient of refractive index of air, $d n / d h$. Here $h$ is height above ground. When the U.S. Standard Atmosphere is considered, it is found that $k_{e}$ is approximately equal to $4 / 3$. This is often referred to as the "four-thirds earth radius model". The refractive index of air, $n$, is a function of air temperature, pressure and humidity, and is usually taken, subject to certain assumptions, as (?),

$$
N=(n-1) \times 10^{6}=77.6 \frac{p}{T}+3.73 \times 10^{5} \frac{e}{T^{2}},
$$

where $p$ is the air pressure (including the water vapor pressure, in $\mathrm{hPa}), e$ is the water vapor pressure $(\mathrm{hPa})$, and $T$ is the air temperature $(\mathrm{K})$. It is convenient to use the quantity $N$, defined as the atmospheric radio refractivity, instead of $n . N$ represents the departure of $n$ from unity in parts per million. $N$ typically has a value of about 300 near the surface and its variation with the height, $d N / d h$, can be considered more conveniently.

The following two equations relate the height above ground, $h$, and the surface range (distance along the earth's surface from radar), $s$, to radar-measurable parameters, the slant path, $r$ and radar elevation angle, $\theta_{e}$ (Doviak and Zrnic, 1993),

$$
\begin{aligned}
& s=a_{e} \sin ^{-1}\left(\frac{r \cos \theta_{e}}{a_{e}+h}\right), \\
& h=\left(r^{2}+a_{e}^{2}+2 r a_{e} \sin \theta_{e}\right)^{1 / 2}-a_{e} .
\end{aligned}
$$

In Doviak and Zrnic (1993), it is also shown that if $r \ll k_{e} a$, and the coordinates $x, y$ and $z$ are related to the radar range, elevation and azimuth coordinates $\left(r, \theta_{e}, \phi\right)$ by,

$$
\begin{aligned}
& x \approx r \cos \theta_{e}^{\prime} \sin \phi, \\
& y \approx r \cos \theta_{e}^{\prime} \cos \phi, \\
& z=h=\left(a_{e}^{2}+r^{2}+2 r k_{e} \sin \theta_{e}^{\prime}\right)^{1 / 2}-k_{e} a,
\end{aligned}
$$

where $\theta_{e}^{\prime}$, the angle between the radar beam and the earth's tangent plane below the data point, is the sum of two terms expressed as the following:

$$
\theta_{e}^{\prime}=\theta_{e}+\tan ^{-1}\left[r \cos \theta_{e} /\left(a_{e}+r \sin \theta_{e}\right)\right] .
$$

From Eq. (5a) and Eq. (5b), one can easily derive the distance along the earth's surface as,

$$
s^{\prime} \approx r \cos \theta_{e}^{\prime} .
$$

Equation (7) is an approximation of the ray path Eq. (3). Equation (5c) uses the effective earth radius beam height Eq. (4).

\section{Variation of refractivity with respect to temperature and dewpoint}

In Eq. (2), the first term on the right hand side is known as the dry term, the second term the moist 
term. The value of radio refractivity $N$ can be computed from measurements of pressure, $p$, temperature, $T$, and water vapor pressure, $e$. In the troposphere the fractional decrease in $p$ with height is larger than that in $T$, so the variation of radio refractivity $N$ with height, $d N / d h$, is usually negative. For the U.S. Standard Atmosphere, $d N / d h$ is about $-39.2 \mathrm{~km}^{-1}$. If $N$ decreases more (less) rapidly with height than the Standard Atmosphere, the beam may be refracted more (less), and in such cases, the height of a target may be overestimated (underestimated) by the fourthirds earth radius model. In an extreme condition, e.g., in the presence of a sharp refractivity gradient of about $-150 \mathrm{~km}^{-1}$ below $100 \mathrm{~m}$ AGL (above ground level), a ray sent at a small positive elevation angle may actually decrease in height with range and eventually strike the earth surface.

Because the air pressure usually makes a rather stable contribution to the variation of $N$, we will only analyze the variation of $N$ with respect to temperature and moisture. The amount of moisture in the air can be expressed in many forms. Four commonly used moisture variables are dewpoint temperature, $T_{\mathrm{d}}$, water vapor pressure, $e$, relative humidity, and specific humidity. To facilitate comparisons with the response to temperature variations, we choose the dewpoint as the moisture variable for our sensitivity study. A commonly used approximate relation between dewpoint and water vapor pressure is Teten's formula (Krishnamurti, 1986):

$$
e=6.11 \exp \frac{\alpha\left(T_{\mathrm{d}}-273.16\right)}{T_{\mathrm{d}}-\beta},
$$

where for water $\alpha=17.26, \beta=35.86$ and for ice $\alpha=21.87, \beta=7.66$. Taking the leading-order variation of Eq. (8) with respect to dewpoint gives

$$
\delta e=e\left[\frac{\alpha(273.16-\beta)}{\left(T_{\mathrm{d}}-\beta\right)^{2}}\right] \delta T,
$$

where $\delta e$ is the variation of water vapor, $e$, and $\delta T_{d}$ is the variation of dewpoint.

By taking the leading-order of variation of the refractivity equation [Eq. (2)] with respect to temperature and water vapor pressure, we have

$$
\begin{aligned}
\delta N= & -\left(\frac{77.6 P}{T^{2}}+\frac{2 \times 3.73 \times 10^{5} e}{T^{3}}\right) \delta T+ \\
& \frac{3.73 \times 10^{5}}{T^{2}} \delta e,
\end{aligned}
$$

where $\delta N$ is the variation of refractivity, and $\delta T$ is the variation of temperature. Substituting Eq. (9) into
Eq. (10), and letting

$$
\begin{aligned}
& A \equiv \frac{\partial N}{\partial T}=-\left(\frac{77.6 p}{T^{2}}+\frac{2 \times 3.73 \times 10^{5}}{T^{3}}\right), \\
& B \equiv \frac{\partial N}{\partial T_{\mathrm{d}}}=\frac{3.73 \times 10^{5}(273.16-\beta) \alpha e}{T^{2}\left(T_{\mathrm{d}}-\beta\right)^{2}},
\end{aligned}
$$

we have

$$
\delta N=A \delta T+B \delta T_{\mathrm{d}}
$$

It is obvious from Eqs. (11) and (12), $A<0$ and $B>0$. These two terms reflect the response of refractivity to temperature and dewpoint variations respectively. Figures $1 \mathrm{a}$ and $1 \mathrm{~b}$ show the variations of $A$ and $B$ as a function of base variables temperature and dewpoint, respectively, within a temperature range of $-10^{\circ} \mathrm{C}$ to $40^{\circ} \mathrm{C}$, and a dewpoint range of $-32^{\circ} \mathrm{C}$ to $40^{\circ} \mathrm{C}$ (or from about $10 \%$ to $100 \%$, in terms of relative humidity) at a constant pressure of $1000 \mathrm{hPa}$. Figure 1c shows the absolute ratio between the variation with dewpoint $(\operatorname{term} B)$ and that of temperature (term $A)$. It is clear from Fig. 1c that refractivity is more sensitive to dewpoint than temperature when the base temperature is high. Note especially that when the base temperature is at or above $30^{\circ} \mathrm{C}$ and the base dewpoint is greater than -16.0 , the variability of refractivity with respect to dewpoint is 5 to 6 times greater in magnitude than with respect to temperature. When the low-level temperature is between $10^{\circ} \mathrm{C}$ to $30^{\circ} \mathrm{C}$, the temperatures typically found in mid-latitudes from spring to early fall, the variability of refractivity with respect to dewpoint is 2 to 4 times greater than to temperature. When the temperature is around $0^{\circ} \mathrm{C}$, the winter situation, the change of refractivity with respect to temperature and moisture variables are of similar magnitudes (see the low-left corner of Figs. 1a and $1 \mathrm{~b})$. Since $A$ and $B$ have opposite signs, the gradient of refractivity is often nearly constant in the upper levels of the atmosphere and during winter, when and where air temperature is low. When the base pressure is set to $700 \mathrm{hPa}$, the pattern of variation is very similar to that shown in Fig. 1, though values of term $A$ and $B$ are slightly smaller (not shown). Therefore, the above discussion is applicable for the entire depth of a typical planetary boundary layer, where the humidity has a significant influence on the atmosphere.

Dividing (13) by $\delta h$, we get

$$
\frac{\delta N}{\delta h}=A \frac{\delta T}{\delta h}+B \frac{\delta T_{\mathrm{d}}}{\delta h} .
$$



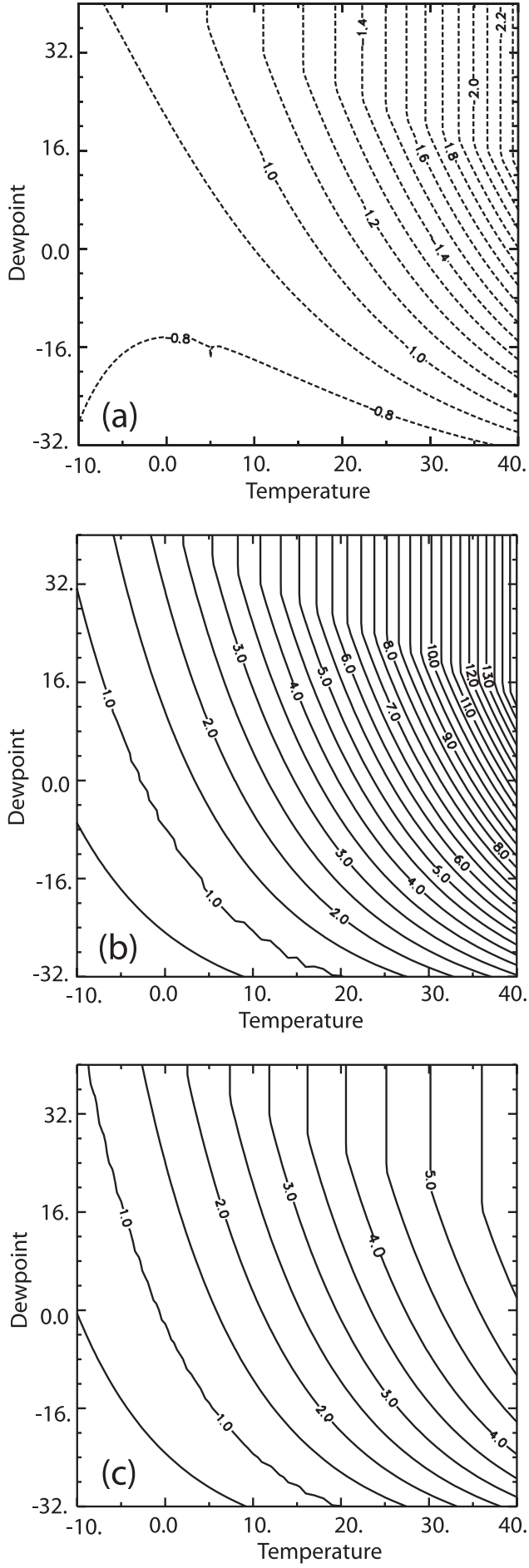

Fig. 1. The variation of refractivity with respect (a) to temperature $\left({ }^{\circ} \mathrm{C}\right)$ indicated by the contours of $A \equiv$ $\partial N / \partial T$ in (11), and (b) to dewpoint $\left({ }^{\circ} \mathrm{C}\right)$ indicated by the contours of $B \equiv \partial N / \partial T_{\mathrm{d}}$ in (12), and (c) the absolute ratio between $B$ and $A$.

Normally, both temperature and dewpoint decrease with the height, i.e., $\delta T / \delta h<0$ and $\delta T_{\mathrm{d}} / \delta h<$
0 . So, the temperature term makes a positive contribution to the rate of decrease in $N$ but the moisture term makes a negative contribution. To satisfy the condition that the decrease in $N$ with height exceeds a critical value (i.e., $\delta N / \delta h<-157 \mathrm{~km}^{-1}$ ), and so that electromagnetic beams are bent toward the surface of the earth, i.e., for them to be trapped, either $\delta T / \delta h$ should be greater than zero, which happens in the inversion layers, or $\delta T_{\mathrm{d}} / \delta h$ should be much less than zero, which happens when a very dry layer overlays a relatively moist layer.

To further quantify our analysis, given a basic state with relative humidity $\mathrm{RH}=60 \%$, temperature $T=17^{\circ} \mathrm{C}$ and pressure $p=1000 \mathrm{hPa}$, we can calculate the values of the other base variables $T_{\mathrm{d}}=11.7^{\circ} \mathrm{C}$, $e=13.7 \mathrm{hPa}$ and $N=328.25$. Substituting these values into Eqs. (11) and (12), we get

$$
A \equiv \frac{\partial N}{\partial T}=-1.34
$$

and

$$
B \equiv \frac{\partial N}{\partial T_{\mathrm{d}}}=4.02 .
$$

These values indicate that a $1^{\circ} \mathrm{C}$ change in temperature causes a 1.34 unit change in refractivity $N$; while a $1^{\circ} \mathrm{C}$ change in dewpoint causes a 4.02 unit change in refractivity. Since variability on the order of few degrees is typical of both temperature and dewpoint in the lower atmosphere, we can therefore say that the radio refractivity is about three times more sensitive to dewpoint than to temperature near the surface for the above typical condition. This point will be further demonstrated in section 5. Among a large number of soundings that we examine in section 5 , many of the most extreme deviations of ray paths from the fourthirds earth model are caused by large vertical moisture gradients; usually when a very dry layer is present above a moist boundary layer. From our discussion, it can also be concluded that it is easier to retrieve moisture variations than temperature from refractivity if it is observed by radars or satellites during the warm season. Weckwerth et al. (2005) showed an interesting result that under most daytime summertime conditions, refractivity from the radar measurements was representative of an atmospheric layer about 250 $\mathrm{m}$ deep layer and could be useful for detecting low-level moisture boundaries.

\section{A stepwise ray tracing method}

In the last two sections, we presented a review on the ray path equations based on the four-thirds earth radius model, and analyzed the variation of radio refractivity with respect to temperature and dewpoint. 
In this section, the influence of different environmental thermodynamic profiles on the radar ray path is examined by using actual observed sounding data. To accurately estimate the radar ray path based on arbitrary sounding data, a stepwise ray tracing method is employed whose steps of calculation are as follows:

(1) Starting from the second range gate from the radar, for each radar measurement, calculate the refractivity $N_{i-1}$ for the previous gate according to Eq. (2) based on the given thermodynamic profile where $i$ is the index of the gates. Calculate the gradient of refractive index according to the differential of Eq. (2) with respect to beam height $h$,

$$
\left.\frac{d n}{d h}\right|_{i-1}=\left.10^{-6} \frac{d N}{d h}\right|_{i-1} .
$$

(2) Calculate $a_{e, i-1}=k_{e, i-1} a$ according to Eq. (1) using the gradient of refractive index from step (1) at the previous gate, $i-1$;

(3) Calculate the angle between the radar beam and the tangent plane below the data point, $\theta_{e, i-1}^{\prime}$ using Eq. (6) for each radar gate;

(4) Finally, calculate the radar beam height $h$ and the surface range $s$ for gate $i$ using formulas

$$
\left\{\begin{array}{c}
h_{i}=h_{i-1}+\Delta r \sin \left(\theta_{e, i-1}^{\prime}\right), \\
s_{i}=s_{i-1}+\Delta r \cos \left(\theta_{e, i-1}^{\prime}\right),
\end{array}\right.
$$

where $\Delta r$ is the gate spacing, which is $250 \mathrm{~m}$ for U.S. operational WSR-88D radial velocity. Variables $h_{i}$ and $s_{i}$ bare the beam height and surface distance for each gate, respectively. Steps (1) through (4) are repeated for successive gates until the last gate of each beam. Note that within this study a single observed sounding is used for each case, thus, we assume that the sounding profile is representative of the vertical structure of the atmosphere within the entire radar range, which may not be true for some cases. For the data assimilation purposes, three dimensional gridded fields of temperature, moisture and pressure can be used to determine the local values of refractivity and further used in ray tracing calculations. The gridded fields can be from the analysis background or from a preliminary analysis that has already incorporated sounding and other non-radar observations.

\section{Ray paths as determined from observed soundings}

In this section, we examine the influence of radio refractivity on the ray path and its climatology in representative geographical regions of the United States by calculating the ray paths for historic sounding data during a six-year period from 1 January 1998 to 31 December 2003 at four locations; namely, Oakland, California (OAK), Key West, Florida (EYW), Dulles Airport, Virginia (IAD) and Topeka, Kansas (TOP). These sites were chosen to represent the West Coast, Tropical Southeast, East Coast and Great Plains regions of the United States, respectively. Qualitycontrolled soundings were obtained from the online database of the NOAA Global Systems Division of the Earth System Research Laboratory.

For each sounding, the radar beam heights at all range gates, for the lowest $0.5^{\circ}$ elevation, are calculated, using the four-thirds earth model and the stepwise ray-tracing method with the actual observed atmospheric profiles. The difference of the beam heights using these two methods is then divided by the corresponding beam width at the corresponding range, assuming a 0.93 degree beam width for the United States WSR-88D network radars. The result can be regarded as relative beam height error, and is relevant because the radar observation is taken to be representative of the volume described by the beam width rather than a point measurement. This metric is chosen because, in relation to the use of the data in numerical models, there is smoothing in the vertical corresponding to the beam shape and thus there are changes in position that are small relative to the beam width and will have a small effect on the integrated forward model result. Table 1 shows the distribution of errors among six error intervals for the locations $50 \mathrm{~km}$ from a hypothetical radar site at the location and elevation of the radiosonde launch site. Among more than 4000 soundings for each site over the 6 year period, we find that, most of the time, the ray paths determined from the four-thirds earth radius model are in good agreement with the stepwise ray tracing method. More than $90 \%$ of the soundings result in relative beam height errors of less than 0.2 . The ray paths determined from the

Table 1. Distribution of relative beam height errors among 6 error intervals for locations $50 \mathrm{~km}$ from the radar site.

\begin{tabular}{cccccccc}
\hline Raob Sites & Obs. No. & \multicolumn{5}{c}{ Error Distributions (\%) } \\
\cline { 3 - 7 } & & {$[0,0.2)$} & {$[0.2,0.4)$} & {$[0.4,0.6)$} & {$[0.6,0.8)$} & {$[0.8,1.0)$} & {$[1.0, \infty]$} \\
\hline OAK & 4234 & 94.31 & 4.65 & 0.92 & 0.12 & 0.00 & 0.00 \\
EYW & 4202 & 94.10 & 5.02 & 0.79 & 0.05 & 0.00 & 0.05 \\
IAD & 4088 & 97.31 & 0.86 & 1.10 & 0.05 & 0.44 & 0.24 \\
TOP & 4253 & 93.63 & 3.74 & 1.27 & 0.75 & 0.19 & 0.42 \\
\hline
\end{tabular}


Table 2. Distribution of relative beam height errors among 6 error intervals for locations $120 \mathrm{~km}$ from the radar site.

\begin{tabular}{cccccccc}
\hline Raob Sites & Obs. No. & \multicolumn{5}{c}{ Error Distributions (\%) } \\
\cline { 3 - 7 } & & {$[0,0.2)$} & {$[0.2,0.4)$} & {$[0.4,0.6)$} & {$[0.6,0.8)$} & {$[0.8,1.0)$} & {$[1.0, \infty]$} \\
\hline OAK & 4234 & 76.17 & 4.82 & 7.58 & 10.01 & 1.42 & 0.00 \\
EYW & 4202 & 71.68 & 11.61 & 7.38 & 8.02 & 1.26 & 0.05 \\
IAD & 4088 & 91.34 & 3.69 & 1.71 & 1.32 & 1.10 & 0.83 \\
TOP & 4253 & 86.93 & 5.48 & 1.98 & 2.66 & 0.59 & 2.37 \\
\hline
\end{tabular}
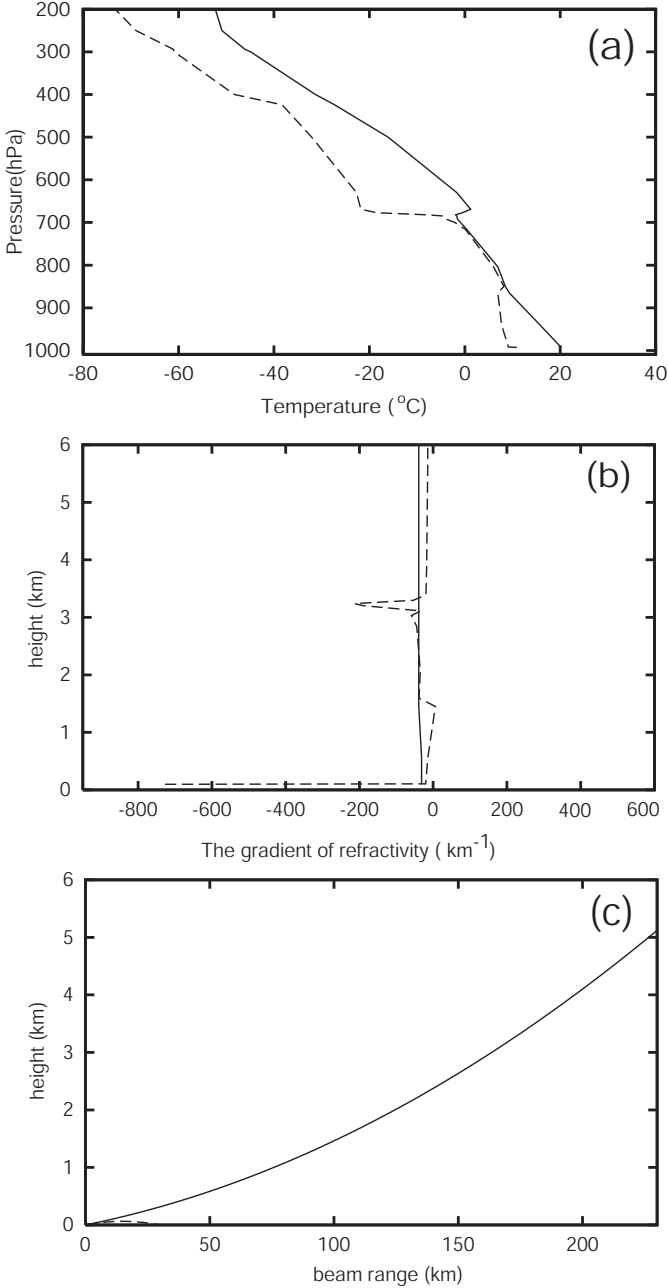

Fig. 2. (a) The temperature (solid) and dewpoint (dashed) profiles, (b) the refractivity gradient profiles $\left(\mathrm{km}^{-1}\right)$ calculated from the U.S. Standard Atmosphere (solid) and from the observed sounding (dashed), and (c) the radar ray paths calculated for the $0.5^{\circ}$ elevation using the Standard Atmosphere (solid) and actual sounding and ray tracing method (dashed, only clear in between 0-50 km), for 0000 UTC 3 May 1999 at Topeka, Kansas (TOP).

four-thirds earth radius model are, on average, more accurate with the soundings from Oakland, California (OAK) than those from soundings of other sites; no relative errors greater than 0.8 are observed with the
Oakland sounding profiles. The ray paths are less accurate with the soundings from Topeka, Kansas, with $0.4 \%$ of soundings having relative beam height errors greater than unity.

Table 2 shows the distribution of errors for the locations $120 \mathrm{~km}$ away from the virtual radar sites. For this distance, over $70 \%$ of soundings result in relative beam height errors of less than 0.2 for OAK and EYW. The number of soundings having beam height errors less than 0.2 is $91.3 \%$ and $86.9 \%$ for IAD and TOP, respectively, which are better than those for OAK and EYW sites. However, the numbers of soundings which result in relative beam height errors of above 1 are larger, at $0.8 \%$ and $2.4 \%$, at IAD and TOP, respectively. As we might expect, range gates further away from the radar sites are more likely to have larger beam height errors using the four-thirds earth radius model due to the accumulation of error over a distance and a greater chance of encountering a layer with an extreme refractivity gradient. Thus, radar data far away from radar sites are more prone to have location errors compared to data closer to the sites.

From Table 2, we also notice that more than $20 \%$ of soundings result in beam height errors of between 0.2 and 0.8 for OAK and EYW, but less than $10 \%$ for IAD and TOP lie in the same range. No soundings from the OAK site and only $0.05 \%$ of soundings from EYW had relative errors greater than 1. However, $0.83 \%$ for IAD and $2.37 \%$ for TOP result in errors of 1 or greater. This indicates that while more soundings from IAD and TOP result in accurate ray path calculations based on the simple model, they also give rise to more cases which have very large relative errors, indicating more variability in the vertical refractivity at these sites.

Figure 2 shows the sounding, refractivity profile and the calculated ray path for one of the worst cases from the TOP site. It is clear that the very strong moisture gradient found in this sounding is responsible for the large vertical refractivity gradient (Figs. 2a, b). The radar beam refracted downward toward the earth surface due to the layer of sharp refractivity gradient below the 1-km level. In this case, the gradient of radio refractivity is largely caused by the vertical variations in humidity. For this site, we also examined 

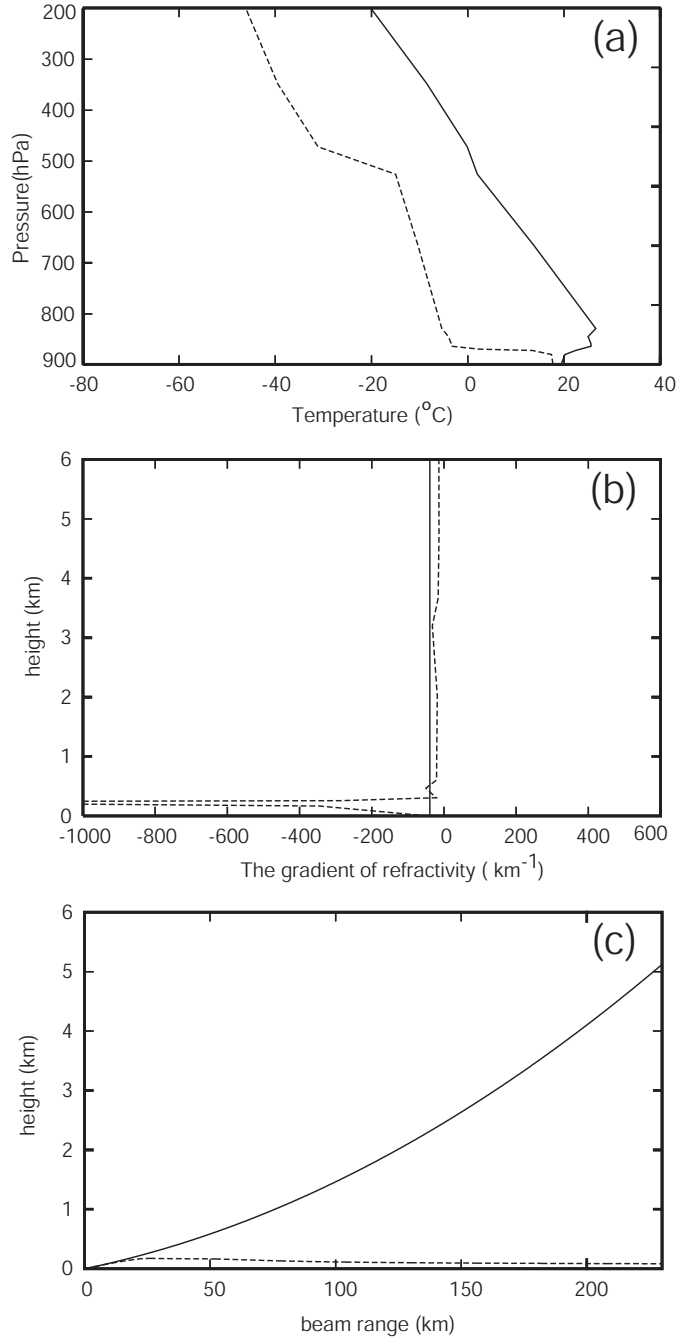

Fig. 3. As Fig. 2, but for 1200 UTC 8 June 2005 at Amarillo, Texas (AMA).

many other cases having large ray path deviations. In most of those cases a large moisture gradient was found at the low levels which caused the beams to be refracted to the ground at a close radar range (as seen in Fig. 2c). Therefore, within the period examined, the beam ducting phenomena occurred more often in the Great Plains and East Coast areas of the U.S. than in the West Coast or Tropical Southeast areas because large moisture gradients near the surface occurred more frequently. In the Great Plains, this situation can be caused by high boundary-layer moisture from local sources or due to advection from the Gulf of Mexico that is overlaid with dry air coming from the Rockies to the west. Similarly for Virginia, dry air advected from the Appalachians, or with a history of subsidence, can often be found above a shallow layer of moist air near the ground with origins from the Atlantic Ocean.
As an illustration of a case that for which the environmental thermodynamic structure had a clear effect on the radar observations, Fig. 3 shows a recent case study for Amarillo, Texas. A large moisture gradient and a temperature inversion (roughly 100 m AGL) are quite pronounced. The calculated beam is trapped in a layer just $100 \mathrm{~m}$ above the ground (Fig. 3c). To show the effect on the radar data in this case, Fig. 4 shows the radar image at 1347 UTC 8 June 2005 from WSR88D radar at Amarillo, Texas (KAMA). The beam might be partially or completely hitting ground targets in places where colors of orange, red or white are found in the figure, as indicated within the area denoted by the circle. In addition, many pixels within the areas showing high reflectivity have been removed by the automated clutter filter (black adjacent to red or white areas).

Suppose that we require that the error in the beam height relative to the beam width be no more than 0.5 for the location estimate to be accurate enough for data assimilation purposes, then we can see from Tables 1 and 2 that the number of soundings which qualify for the use of the four-thirds earth radius model for the ray path calculation is well above $90 \%$. However, beam ducting and strong departures from the four-thirds earth model do occur at small percentages, especially in the Great Plains and Eastern United States. Some of these could be found in situations preceding severe weather events - situations for which the WSR-88D radar data are most needed. Most of these phenomena are caused by large moisture gradients in the lower atmosphere. For this reason, quality control and data assimilation systems should check for such situations, and, when present, use the more accurate ray-trace method for beam height calculation and/or discard low-level data that may be contaminated by ground targets due to beam ducting and/or inaccurate height determination.

\section{Summary and conclusions}

Radar ray path equations are used to determine the physical location of each radar measurement for data display, radar-data based automated detection algorithms, quality control and data assimilation. To best use radar data, the accuracy of ray path calculations and the assumptions involved need to be examined thoroughly to see if significant deviations from the typically used Standard-Atmosphere-based four-thirds effective earth radius model occur frequently. Such large deviations occur when a strong temperature inversion and/or large vertical moisture gradient exist. In this study, we first analyzed the variation of radio refractivity with respect to atmospheric temperature 


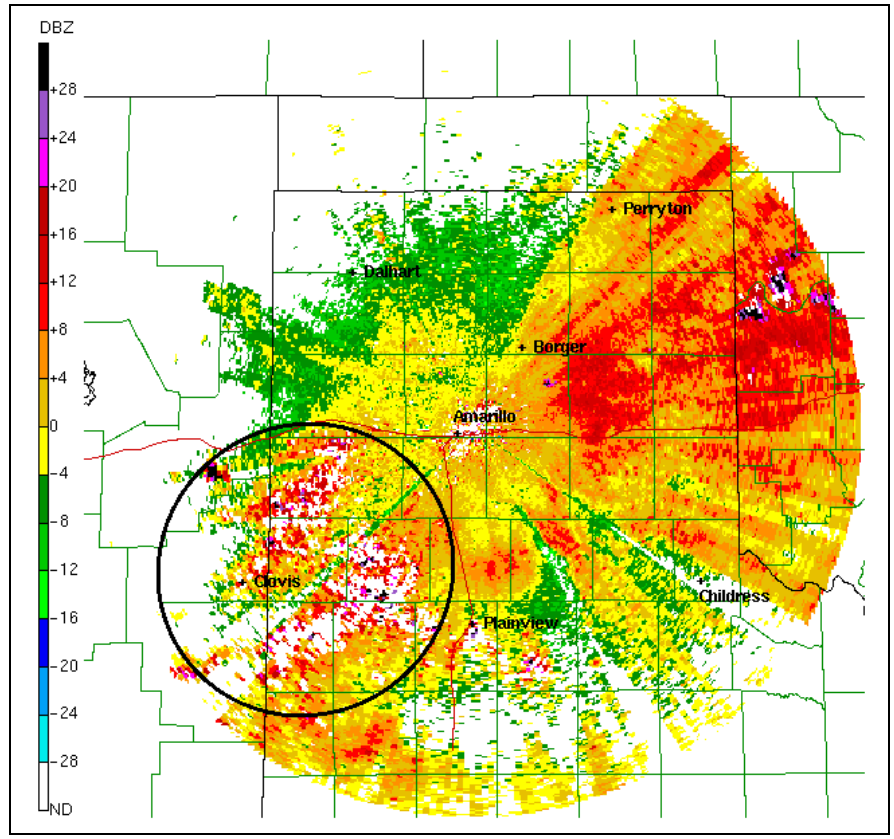

Fig. 4. Radar reflectivity (dBZ) image at 1347 UTC 8 June 2005 for KAMA at Amarillo, Texas.

and moisture. It is shown that radio refractivity gradient is more sensitive to the moisture than to temperature; therefore moisture has a more significant influence on the radar ray path calculation than temperature.

To accurately calculate the radar ray path based on general sounding profiles, a stepwise ray tracing program is developed. The influence of atmospheric refractivity on the ray path is examined using a large number of historic soundings from four sites representing different geographical regions of the United States. For the soundings examined, 90\% result in small relative beam height errors when calculated using the standard-atmosphere-based four-thirds earth radius model and only a small fraction of ray paths thus calculated diverge significantly from those calculated based on true soundings using the ray tracing method. But these small fractions of deviations could occur more often in situations preceding severe weather. For many of the problematic cases examined, the vertical moisture gradient is found to be a more significant contributor. The results of this paper may provide a useful guidance to radar data quality control, as well as the assimilation of radar data into numerical weather prediction models.

Acknowledgements. This work was supported by U. S. NSF Grant Nos. ATM-0331756, ATM-0331594, ATM-0530814 and EEC-0313747, and by DOT-FAA Grant NA17RJ1227-01. The first and third authors were also supported partially by the National Natural Science Foundation of China under Grant Nos. 40620120437 and 40505022 .

\section{REFERENCES}

Bean, B. R., and E. J. Dutton, 1968: Radio Meteorology. Dover Publication, 435pp.

Bech, J., B. Codina, J. Lorente, and D. Bebbington, 2003: The sensitivity of single polarization weather radar beam blockage correction to variability in the vertical refractivity gradient. J. Atmos. Oceanic Technol., 20, 845-855.

Brewster, K. A., 2003: Phase-correcting data assimilation and application to storm scale numerical weather prediction. Part II: Application to a severe storm outbreak. Mon. Wea. Rev., 131, 493-507.

Crook, N. A., and J. Sun, 2002: Assimilating radar, surface, and profiler data for the Sydney 2000 Forecast Demonstration Project. J. Atmos. Oceanic Technol., 19, 888-898.

Crum, T. D., and R. L. Albert, 1993: The WSR-88D and the WSR-88D operational support facility. Bull. Amer. Meteor. Soc., 74, 1669-1687.

Crum, T. D., R. E. Saffle, and J. W. Wilson, 1998: An update on the NEXRAD Program and future WSR-88D support to operations. Wea. Forecasting, 13, 253262.

Doviak, R. J., and D. S. Zrnic, 1993: Doppler Radar and Weather Observations. Academic Press, 2nd ed., 562pp.

Droegemeier, K. K., 1990: Toward a science of storm- 
scale prediction. Preprint, 16th Conf. on Severe Local Storms, Kananaskis Park, Alberta, Canada, Amer. Meteor. Soc., 256-262.

Droegemeier, K. K., 1997: The numerical prediction of thunderstorms: Challenges, potential benefits, and results from real time operational tests. WMO Bulletin, 46, 324-336.

Gao, J., M. Xue, Z. Wang, and K. K. Droegemeier, 1998: The initial condition and explicit prediction of convection using ARPS forward assimilation and adjoint methods with WSR-88D data. Preprints, 12th Conf. Num. Wea. Pred., Phoenix, AZ, Amer. Meteor. Soc., 176-178.

Gao, J., M. Xue, K. Brewster, and K. K. Droegemeier 2004: A three-dimensional variational data assimilation method with recursive filter for single-Doppler radar. J. Atmos. Oceanic Technol., 21, 457-469.

Gao, J., K. Brewster, and M. Xue, 2006: A comparison of the radar ray path equation and approximations for use in radar data assimilation. Adv. Atmos. Sci., 23(2), 190-198, 10.1007/s00376-006-0190-3.

$\mathrm{Hu}, \mathrm{M} ., \mathrm{M}$. Xue, and K. Brewster, 2006a: 3DVAR and cloud analysis with WSR-88D level-II data for the prediction of Fort Worth tornadic thunderstorms. Part I: Cloud analysis and its impact. Mon. Wea. Rev., 134, 675-698.

$\mathrm{Hu}$, M., M. Xue, J. Gao, and K. Brewster, 2006b: 3DVAR and cloud analysis with WSR-88D level-II data for the prediction of Fort Worth tornadic thunderstorms. Part II: Impact of radial velocity analysis via 3DVAR. Mon. Wea. Rev., 134, 699-721.

Krishnamurti, T. N., 1986: Workbook on numerical weather prediction for the tropics for the training of class I and class II meteorological personnel. Published by World Meteorological Organization, No. 669, Geneva, Switzerland.

Lilly, D. K., 1990: Numerical prediction of thunderstorms-Has its time come? Quart. J. Roy. Meteor. Soc., 116, 779-798.

Moszkowicz, S., G. J. Ciach, and W. F. Krajewski, 1994:
Statistical detection of anomalous propagation in radar reflectivity patterns. J. Atmos. Oceanic Technol., 11, 1026-1034.

Pamment, J. A., and B. J. Conway, 1998: Objective Identification of Echoes Due to Anomalous Propagation in Weather Radar Data. J. Atmos. Oceanic Technol., 15, 98-113.

Serafin, R. J., and J. W. Wilson, 2000: Operational weather radar in the United States: Progress and opportunity. Bull. Amer. Meteor. Soc., 81, 501-518.

Shapiro, A., P. Robinson, J. Wurman, and J. Gao, 2003: Single-Doppler velocity retrieval with rapidscan radar data. J. Atmos. Oceanic. Technol., 20, 1758-1775.

Sun, J., D. W. Flicker, and D. K. Lilly, 1991: Recovery of three-dimensional wind and temperature fields from simulated single-Doppler radar data. J. Atmos. Sci., 48, 876-890.

Sun, J., and N. A. Crook, 2001: Real-time low-level wind and temperature analysis using single WSR88D data. Wea. Forecasting, 16, 117-132.

Weckwerth, T. M., C. R. Pettet, F. Fabry, S. Park, M. A., LeMone, and J. W. Wilson, 2005: Radar refractivity retrieval: Validation and application to short-term forecasting. J. Appl. Meteor., 44, 285-300.

Weygandt, S. S., A. Shapiro, and K. K. Droegemeier, 2002a: Retrieval of initial forecast fields from singleDoppler observations of a supercell thunderstorm. Part I: Single-Doppler velocity retrieval. Mon. Wea. Rev., 130, 433-453.

Weygandt, S. S., A. Shapiro, and K. K. Droegemeier, 2002b: Retrieval of initial forecast fields from singleDoppler observations of a supercell thunderstorm. Part II: Thermodynamic retrieval and numerical prediction. Mon. Wea. Rev., 130, 454-476.

Xue, M., D.-H. Wang, J. Gao, K. Brewster, and K. K. Droegemeier, 2003: The Advanced Regional Prediction System (ARPS), storm-scale numerical weather prediction and data assimilation. Meteorology and Atmospheric Physics, 82, 139-170. 